\title{
Addressing the Opioid Crisis: Community Partnerships in Primary Care
}

Jamie Weinand, MD | Athena Huckaby, MPH | Olivia Chavez, RN | Ramona Sharma, MD | Jeanette Lara, DO | Laura Leija, MD | Christopher Morriss, MD | Shawn Rowland, MD | Davena Norris, PharmD | Minerva Medrano de Ramirez, MD | Salvador Adame-Zambrano, MD | John Andazola, MD I Ivan De La Rosa, PhD, LMSW

PRiMER. 2019;3:20.

Published: 9/13/2019 | DOI: 10.22454/PRiMER.2019.649767

\section{Abstract}

Introduction: New Mexico is currently ranked 17th in the United States for drug overdose death rates. Our project seeks to decrease opioid overdose deaths in a community by increasing the number of patients with naloxone in a local family medicine residency clinic.

Methods: We developed a protocol wherein providers asked patients at risk of opioid overdose about naloxone access. Free naloxone was distributed in partner with the county health department, accompanied by teaching of use. We reviewed patient encounters during a 45-day control and study period to measure naloxone possession among patients at risk.

Results: Nearly two-thirds of patients at risk of opioid overdose had no naloxone. A standardized protocol implemented to distribute an opioid reversal agent doubled naloxone prescribed by providers at visits (10.3\%) compared to a control period (4.3\%), but lacked statistical significance.

Conclusion: Patients in a family medicine residency clinic who were at risk of opioid overdose overwhelmingly did not have naloxone, and a standardized protocol with a community-based partnership increased access to naloxone. Further project data will have implications for ongoing naloxone distribution programs in primary care.

\section{Introduction}

Drug overdose is now the leading cause of death for Americans under age 50 years. ${ }^{1}$ New Mexico is ranked 17th nationwide for overdose deaths, and New Mexico overdose deaths tripled between 1990 and 2015 (Figure 1). ${ }^{2}$

Current evidence shows that naloxone distribution reduces opioid-related overdose deaths and is cost effective. ${ }^{3-7}$ The American Academy of Family Physicians recommends family physicians prescribe naloxone, an opioid reversal agent, to patients at high risk of overdose and support community naloxone distribution programs. ${ }^{8}$

This project seeks to decrease opioid overdose deaths in a community by increasing the number of people with naloxone, and assesses effectiveness of a brief intervention that can be performed in a typical primary care clinic with implications for harm reduction at the community level. 
A group of family medicine residents located in New Mexico created a quality improvement project to address the opioid overdose crisis within their clinic in August 2018. They developed a standardized protocol (Appendix 1) wherein providers asked patients about access to personal naloxone if identified to be at risk of opioid overdose (defined as the self-reported use of illicit opioids during that visit, or presence of a prescription opioid in the visit's medication reconciliation). This project was implemented prior to the June 2019 New Mexico mandate of coprescription of naloxone with opioid prescriptions for 5 days or more. ${ }^{9}$ The clinic partnered with the local health department to distribute free naloxone to patients at risk of opioid overdose. Intranasal naloxone was given to the patients during their clinic appointments with instruction regarding its use from the provider. Patients who received naloxone were given a unique identifier to identify receipt of naloxone for the first or second time, thereby identifying possible overdose. Patients were able to return to the clinic outside of appointments to refill naloxone if used. The total morphine milligram equivalent (MME) dose of the patient and the presence or absence of naloxone was flagged in the chart during data analysis to inform providers in subsequent visits. Naloxone kits were provided to patients regardless of MME. In addition to providing naloxone to at-risk patients, it was also available by request. Prior to this community partnership, patients were prescribed naloxone electronically to their pharmacy, with cost varying up to $\$ 150 .{ }^{10}$ We reviewed all patient encounters during a 45 -day control and investigational period. All charts of patients seen in the time frame were included, excluding pediatric patients. $\chi^{2}$ analyses examined the association between the periods of chart review and naloxone receipt. Our institution's institutional review board exempted the study.

\section{Results}

Patient encounters in the control and investigational periods totaled 1,852 and 1,814, respectively (Figure 2). During the control period, $8.1 \%$ of patient encounters identified a patient at elevated risk of opioid overdose. In the investigational period, $10.4 \%$ of encounters identified a patient at risk.

Among patients at risk for opioid overdose, $61.7 \%$ of control group and $61.9 \%$ of investigational group patients did not have naloxone in their possession as they arrived for that visit; $4.3 \%$ of control group and $10.3 \%$ of investigational group patients were given naloxone at the visit. No patients declined naloxone, and kits were consistently available.

Independence of associations were tested by performing a series of $\chi^{2}$ analyses. Among the experimental and control groups, 129 patients previously possessed naloxone and 209 patients did not (Table 1). These frequencies were not significantly different, $\chi^{2}(1, \mathrm{~N}=338)=.001, P=.976$. Of 209 patients who did not possess naloxone, 193 did not receive naloxone and 16 received naloxone at the visit. These frequencies were not significantly different, $\chi^{2}(1$, $\mathrm{N}=209)=2.54, P=.111$, Cramer $\mathrm{V}=.11, n s$. The odds ratio for patients receiving naloxone at the visit during investigational compared to control period was 2.51 (95\% $\mathrm{Cl} 0.78-8.07)$.

During the investigational period, one patient required a refill of their naloxone outside of an appointment. Because this project implemented both chart review of scheduled appointments and a protocol to distribute naloxone available to patients outside of appointments, this refill was not captured by chart review but was identified by nursing staff who provided the refill.

\section{Conclusions}

Patients in a family medicine residency clinic who were at risk of opioid overdose overwhelmingly did not have naloxone, and a standardized protocol with a community-based partnership increased access to naloxone. Although not significant, patients during the investigative review period were 2.5 times more likely to receive a new naloxone kit. Within both the control and investigational periods, the majority of patients did not receive naloxone; the provider either forgot to offer the naloxone kit or did not follow the protocol. The project also identified a relatively high rate of naloxone possession prior to the intervention. New Mexico has distributed naloxone in the community since 2001 
with a saturation model, and a mandate to distribute to medication-assisted treatment patients for those with buprenorphine or methadone prescriptions. ${ }^{11-12}$

Important barriers to distributing naloxone were encountered. First, providers identified that naloxone distribution is still not routine in their workflow when the visit is not for pain management. Secondly, some patients were reluctant to accept naloxone due to stigma, an ongoing challenge to overcome. Clinic providers aimed to actively listen to patients while withholding judgement. For this reason, patients requesting a naloxone refill were given them without questions regarding the event, in order not to deter reporting due to shame surrounding an overdose.

This study had several limitations. First, we identified at-risk patients by self-reported use of illicit opioids, which may have missed patients due to potential underreporting secondary to stigma. Second, while the quality improvement project showed a trend toward increase in naloxone prescription, this difference was not statistically significant, likely due to small sample size. This trend may be significant if the duration of the investigation and the number of visits were increased.

Further study of this intervention will identify more ways to increase naloxone distribution. One area identified for improvement is utilizing the electronic medical record to integrate naloxone distribution into workflows. Action in these areas may further increase naloxone distribution throughout the community.

\section{Tables and Figures}

Figure 1: Deaths Due to Drug Overdose by Year, New Mexico and United States, 1990-2016²

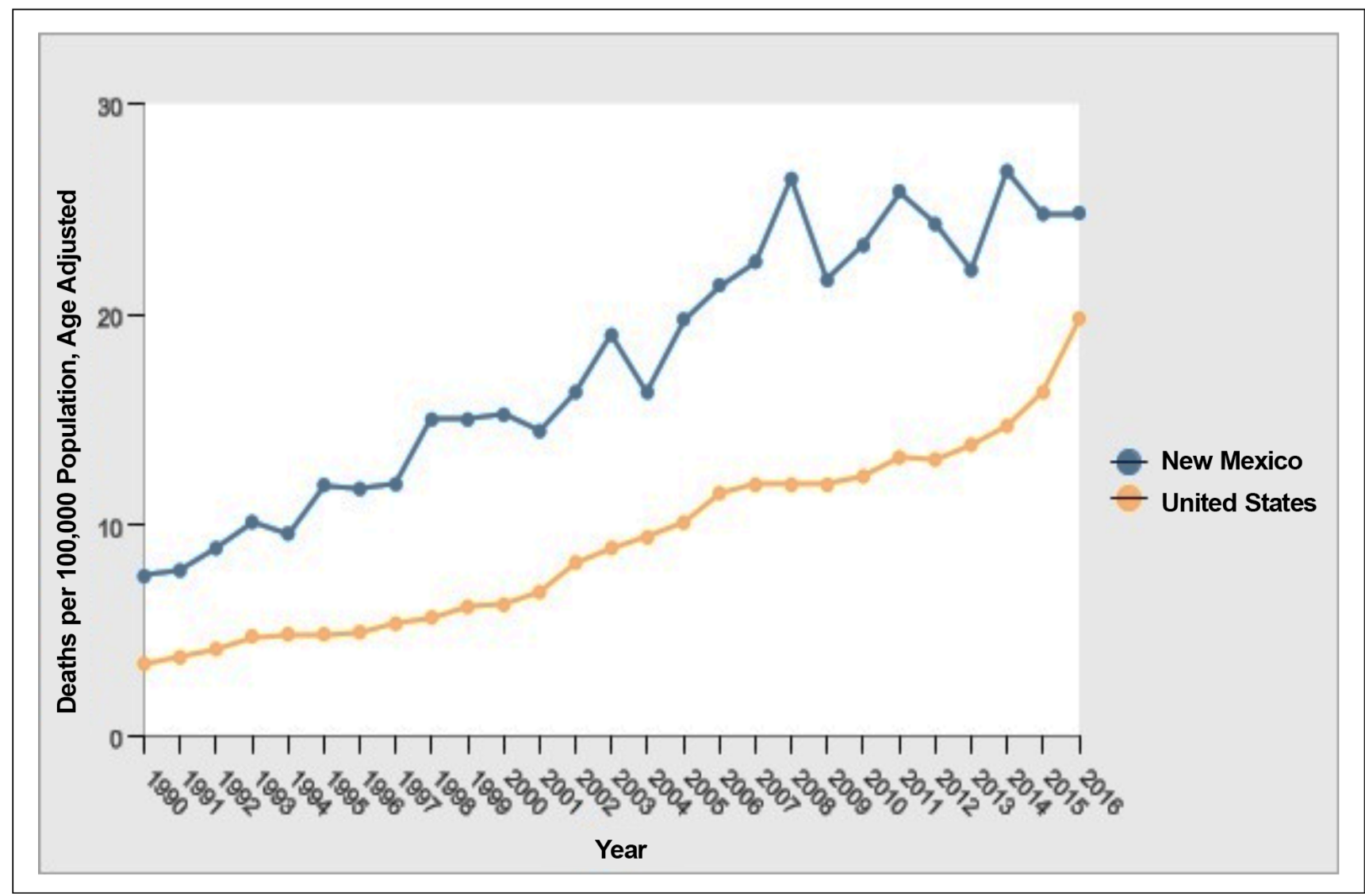


Figure 2: Results: Patient Encounters of the Control and Investigational Periods

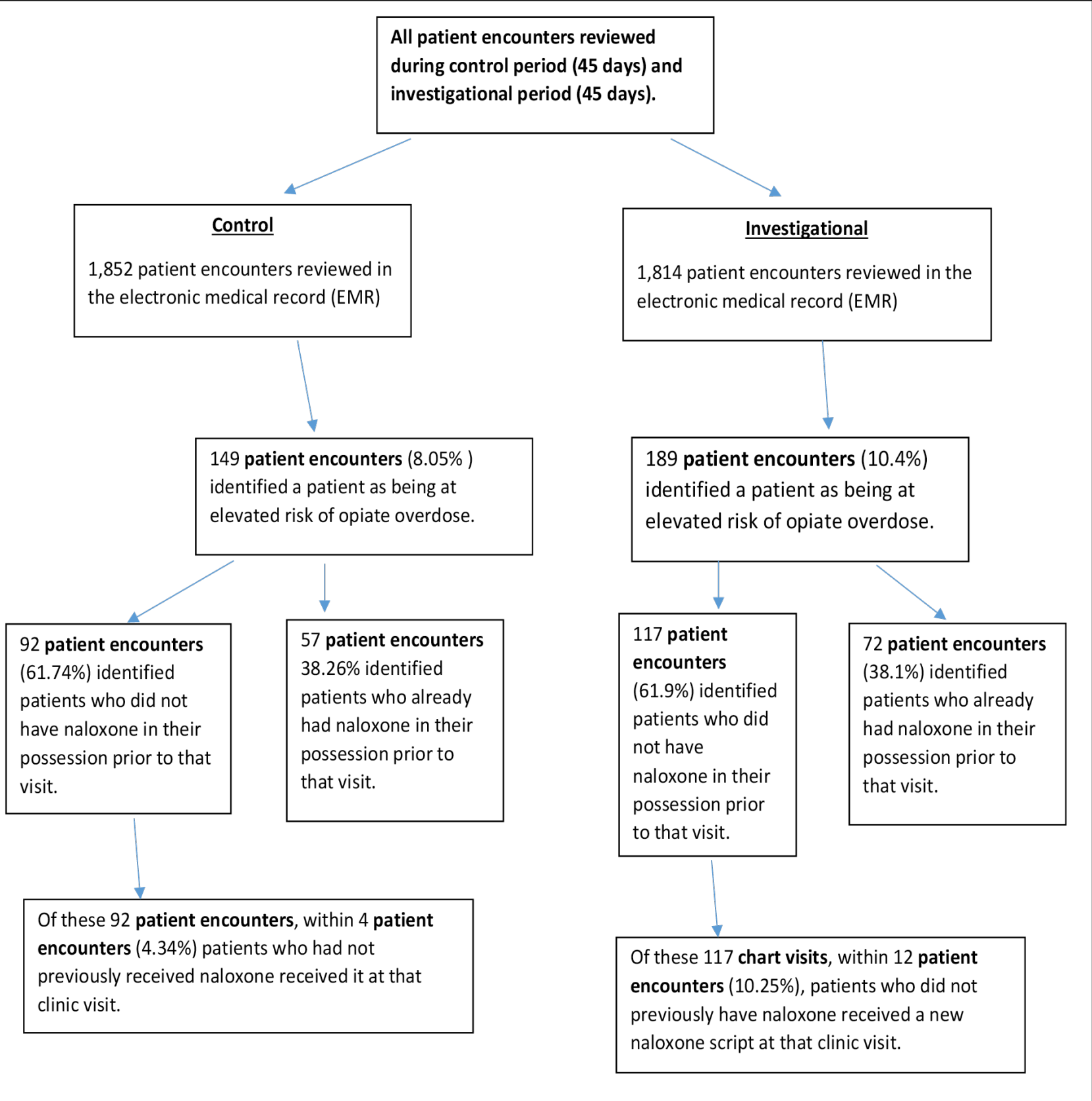

Table 1: Receipt of New Naloxone Script and Period of Chart Review for 209 Patients

\begin{tabular}{|c|c|c|c|c|c|c|}
\hline \multirow[b]{2}{*}{$\begin{array}{l}\text { Received New } \\
\text { Naloxone Script }\end{array}$} & \multicolumn{2}{|c|}{$\begin{array}{l}\text { Control Period } \\
(n=92)\end{array}$} & \multicolumn{2}{|c|}{$\begin{array}{l}\text { Investigative Period } \\
\qquad(n=117)\end{array}$} & \multirow[b]{2}{*}{$\chi^{2}(d i f)$} & \multirow[b]{2}{*}{$\boldsymbol{P}$} \\
\hline & No. & $\%$ & No. & $\%$ & & \\
\hline Yes $(n=16)$ & 4 & 4.5 & 12 & 10.3 & $2.54(1)$ & .111 \\
\hline No $(n=193)$ & 88 & 95.7 & 105 & 89.7 & & \\
\hline
\end{tabular}

Cramer V=.110; OR=2.51 (95\% Cl=.78-8.07).

Note: Column percentage reported.

\section{Acknowledgments}

Financial Support: The Southern New Mexico Family Medicine Residency Program at Memorial Medical Center-the 
clinic site for this project-did not receive any funding. Athena Huckabee, overdose prevention coordinator for the Doña Ana County Health and Human Services Department received a Substance Abuse and Mental Health Services Administration (SAMHSA) grant through the Office of Substance Abuse Prevention (OSAP) at the state level, and the Doña Ana County Health and Human Services Department was selected as a site. This funded the free naloxone that was distributed throughout the community, including with our project. Apart from the free naloxone distributed at our site, there was no other benefit gained from this grant or the Doña Ana County Health and Human Services Department.

Presentations: This study was presented as a "Work in Progress" at the 2019 Society of Teachers of Family Medicine Annual Spring Conference, April 27, 2019, Toronto, ON, Canada.

\section{Corresponding Author}

Jamie Weinand, MD

2450 S. Telshor Blvd, Las Cruces, NM 88011. 520-850-4066. Fax: 575-521-5376.

jamie.d.weinand@gmail.com

\section{Author Affiliations}

Jamie Weinand, MD - Memorial Medical Center, Southern New Mexico Family Medicine Residency Program, Las Cruces, NM

Athena Huckaby, MPH - Doña Ana County Health and Human Services, Las Cruces, NM

Olivia Chavez, RN - Memorial Medical Center, Southern New Mexico Family Medicine Residency Program, Las Cruces, NM

Ramona Sharma, MD - Memorial Medical Center, Southern New Mexico Family Medicine Residency Program, Las Cruces, NM

Jeanette Lara, DO - Memorial Medical Center, Southern New Mexico Family Medicine Residency Program, Las Cruces, NM

Laura Leija, MD - Memorial Medical Center, Southern New Mexico Family Medicine Residency Program, Las Cruces, NM

Christopher Morriss, MD - Memorial Medical Center, Southern New Mexico Family Medicine Residency Program, Las Cruces, NM

Shawn Rowland, MD - Memorial Medical Center, Southern New Mexico Family Medicine Residency Program, Las Cruces, NM

Davena Norris, PharmD - Memorial Medical Center, Southern New Mexico Family Medicine Residency Program, Las Cruces, NM

Minerva Medrano de Ramirez, MD - Memorial Medical Center, Southern New Mexico Family Medicine Residency Program, Las Cruces, NM

Salvador Adame-Zambrano, MD - Memorial Medical Center, Southern New Mexico Family Medicine Residency Program, Las Cruces, NM

John Andazola, MD - Memorial Medical Center, Southern New Mexico Family Medicine Residency Program, Las Cruces, NM

Ivan De La Rosa, PhD, LMSW - Memorial Medical Center, Southern New Mexico Family Medicine Residency Program, Las Cruces, NM I and New Mexico State University, School of Social Work, Las Cruces, NM

\section{References}

1. Centers for Disease Control and Prevention, National Center for Health Statistics. Underlying Cause of Death 1999-2016. CDC WONDER Online Database. http://wonder.cdc.gov/ucd-icd10.html. Published December, 2017. Accessed November 20, 2018.

2. New Mexico's Indicator-Based Information System for Public Health. Complete Health Indicator Report of 
Drug Overdose Deaths. https://ibis.health.state.nm.us/indicator/complete_profile/DrugOverdoseDth.html. Published February, 2018. Accessed November 20, 2018.

3. Walley AY, Xuan Z, Hackman HH, et al. Opioid overdose rates and implementation of overdose education and nasal naloxone distribution in Massachusetts: interrupted time series analysis. BMJ. 2013;346(jan30 5):f174. https://doi.org/10.1136/bmj.f174

4. Coffin PO, Sullivan SD. Cost-effectiveness of distributing naloxone to heroin users for lay overdose reversal in Russian cities. J Med Econ. 2013;16(8):1051-1060. https://doi.org/10.3111/13696998.2013.811080

5. Langham S, Wright A, Kenworthy J, Grieve R, Dunlop WCN. Cost-effectiveness of take-home naloxone for the prevention of overdose fatalities among heroin users in the United Kingdom. Value Health. 2018;21(4):407-415. https://doi.org/10.1016/j.jval.2017.07.014

6. Penm J, MacKinnon NJ, Boone JM, Ciaccia A, McNamee C, Winstanley EL. Strategies and policies to address the opioid epidemic: a case study of Ohio. J Am Pharm Assoc (2003). 2017;57(2S):S148-S153. https://doi.org /10.1016/j.japh.2017.01.001

7. McDonald R, Strang J. Are take-home naloxone programmes effective? Systematic review utilizing application of the Bradford Hill criteria. Addiction. 2016;111(7):1177-1187. https://doi.org/10.1111/add.13326

8. American Academy of Family Physicians.Chronic pain management and opioid misuse: a public health concern. http://www.aafp.org/about/policies/all/pain-management-opioid.html. Published July, 2012. Accessed December 18, 2018.

9. The Legislature of the State of New Mexico. 54th Legislature, 1st Session Laws 2019. Chapter 126. Senate Bill 221. https://nmlegis.gov/Sessions/19\%20Regular/final/SB0221.pdf. Accessed June 30, 2019.

10. Gupta R, Shah ND, Ross JS. The rising price of naloxone - risks to efforts to stem overdose deaths. N Engl J Med. 2016;375(23):2213-2215. https://doi.org/10.1056/NEJMp1609578

11. Legal interventions to reduce overdose mortality: naloxone access and overdose Good Samaritan Laws. The Network for Public Health Law. https://www.leg.state.nv.us/interim/77th2013/Committee/StatCom /ACAJ/Other/1-May-2014/HolmesSummaryofStateOverdoseLawsJuly2013.pdf. July 2013. Accessed May 23, 2019.

12. The Legislature of the State of New Mexico. 53rd Legislature, 1st Session Laws 2017 Chapter 59. House Health and Human Services Committee Substitute for House Bill 370. http://www.sos.state.nm.us/uploads /files/CH59-HB370-2017.pdf. Published April 6, 2017. Accessed May 23, 2019.

Copyright $\odot 2019$ by the Society of Teachers of Family Medicine 